\title{
BMJ Open Biomarker Research in ADHD: the Impact of Nutrition (BRAIN) - study protocol of an open-label trial to investigate the mechanisms underlying the effects of a few-foods diet on ADHD symptoms in children
}

Tim Stobernack, ${ }^{1}$ Stefan P W de Vries, ${ }^{1}$ Rob Rodrigues Pereira,${ }^{2}$ Lidy M Pelsser, ${ }^{3}$ Cajo J F ter Braak, ${ }^{4}$ Esther Aarts, ${ }^{5}$ Peter van Baarlen, ${ }^{1}$ Michiel Kleerebezem, ${ }^{1}$ Klaas Frankena, ${ }^{6}$ Saartje Hontelez (D) ${ }^{1}$

To cite: Stobernack T, de Vries SPW, Rodrigues Pereira R, et al. Biomarker Research in ADHD: the Impact of Nutrition (BRAIN) - study protocol of an open-label trial to investigate the mechanisms underlying the effects of a few-foods diet on ADHD symptoms in children. BMJ Open 2019;9:e029422. doi:10.1136/ bmjopen-2019-029422

- Prepublication history for this paper is available online. To view these files, please visit the journal online (http://dx.doi. org/10.1136/bmjopen-2019029422).

Received 25 January 2019 Revised 02 August 2019 Accepted 12 September 2019

Check for updates

(C) Author(s) (or their employer(s)) 2019. Re-use permitted under CC BY-NC. No commercial re-use. See rights and permissions. Published by BMJ.

For numbered affiliations see end of article.

Correspondence to Dr Saartje Hontelez; ADHD@wur.nl

\section{ABSTRACT}

Introduction Attention deficit hyperactivity disorder (ADHD) is the most common childhood behavioural disorder, causing significant impediment to a child's development. It is a complex disorder with numerous contributing (epi)genetic and environmental factors. Currently, treatment consists of behavioural and pharmacological therapy. However, ADHD medication is associated with several side effects, and concerns about long-term effects and efficacy exist. Therefore, there is considerable interest in the development of alternative treatment options. Double-blind research investigating the effects of a few-foods diet (FFD) has demonstrated a significant decrease in ADHD symptoms following an FFD. However, an FFD requires a considerable effort of both child and parents, limiting its applicability as a general ADHD treatment. To make FFD intervention less challenging or potentially obsolete, we need to understand how, and in which children, an FFD affects ADHD behaviour and, consequently, the child's well-being. We hypothesise that an FFD affects brain function, and that the nutritional impact on ADHD is effectuated by a complex interplay between the microbiota, gut and brain, that is, the microbiota-gut-brain axis.

Methods and analysis The Biomarker Research in ADHD: the Impact of Nutrition (BRAIN) study is an open-label trial with researchers blinded to changes in ADHD symptoms during sample processing and initial data analyses.

Ethics and dissemination The Medical Research and Ethics Committee of Wageningen University has approved this study (NL63851.081.17, application 17/24). Results will be disseminated through peer-reviewed journal publications, conference presentations, (social) media and the BRAIN study website. A summary of the findings will be provided to the participants.

Trial registration number NCT03440346.

Study dates Collection of primary outcome data started in March 2018 and will be ongoing until 100 children have participated in the study. Sample data analysis will start after all samples have been collected.

\section{Strengths and limitations of this study}

- Using unbiased, high-resolution brain imaging and molecular analyses of the microbiota-gut-brain (MGB) axis, the Biomarker Research in ADHD: the Impact of Nutrition (BRAIN) study aims to unravel the mechanism(s) that underlie favourable responses of children with attention deficit hyperactivity disorder (ADHD) to a few-foods diet (FFD).

- Behavioural changes will be assessed using three independent methods, that is, (1) functional MRI to assess brain activity, (2) a quantitative behaviour test and (3) standardised questionnaires.

- The effects of an FFD on the biologically relevant catecholamine neurotransmitter system and related brain regions are evaluated, while multiomics data of MGB axis parameters will allow for an unbiased approach to identify other candidate molecules that may (co)determine FFD effects.

- Identification of baseline molecular signatures (ie, findings prior to intervention) that can predict whether a participant belongs to the FFD responder or non-responder group may lead to the discovery of biomarkers predicting FFD efficacy in future participants.

- To increase the probability of finding potential mechanism(s) and biomarkers, the BRAIN study requires a relatively homogeneous study population.

\section{INTRODUCTION}

Attention deficit hyperactivity disorder (ADHD) affects $6 \%$ of all children worldwide $^{1}$ and is characterised by inattentive, hyperactive and/or impulsive behaviour. ${ }^{2}$ The majority of children with ADHD are also diagnosed with other psychiatric disorders, including oppositional defiant disorder (ODD), conduct disorder, and autism 
spectrum disorder (ASD).$^{3-5}$ ADHD can cause long-term impairment and often persists into adulthood. ${ }^{6}$

ADHD is considered a complex multifactorial disorder with numerous contributing (epi)genetic and environmental factors. Heritability $\left(\mathrm{h}^{2}\right)$ of the disorder is estimated at $0.75-0.91^{7}$ and many genetic variants, often related to neurotransmission and neurodevelopment, ${ }^{89}$ are associated with an increased risk of developing ADHD. ${ }^{10}{ }^{11}$ Epigenetic regulation of gene activity, such as differential DNA methylation of genes related to neurotransmission, ${ }^{12}$ neurodevelopment and peroxisomal processes, is also associated with ADHD. ${ }^{13}$ Environmental risk factors of ADHD include pre, peri and postnatal factors, such as prematurity, low birth weight, in utero exposure to smoking, alcohol and drugs, psychosocial conditions and diet. ${ }^{14} 15$ A combination of (epi) genetic factors may underlie the susceptibility to environmental factors for triggering ADHD,${ }^{15}$ however, the exact aetiology of ADHD remains unknown.

Currently, ADHD treatment predominantly consists of psychoeducation, behavioural therapy and medication. ${ }^{16}$ Drug treatment is deemed superior for children with severe ADHD impairment. ${ }^{16}$ Methylphenidate (MPH), a dopamine-noradrenaline reuptake inhibitor, is the most prescribed drug with positive behavioural effects in children with ADHD. ${ }^{17}$ However, the studies that have investigated the effect of MPH had a risk of bias and lowquality outcomes. ${ }^{17}$ In addition, $25 \%$ of children taking MPH suffer from side effects, ${ }^{17}$ complete normalisation of behaviour is rare, and non-adherence due to concerns about long-term effects is common. ${ }^{18-23}$ Furthermore, MPH is not effective 24 hours a day; when the medication has worn off, symptoms return, sometimes even with increased intensity. ${ }^{24}$ Therefore, novel therapies, aimed to intervene on the underlying causes or triggers of ADHD, are needed.

One of these novel therapies might be dietary treatment. Meta-analysis has demonstrated that elimination of many foods and additives, the so-called few-foods diet (FFD), substantially reduced ADHD symptoms. ${ }^{25}$ During an FFD, children follow a restricted diet for several weeks, in which they consume only a few types of food (eg, rice, meat, vegetables, pear and water), initially complemented with foods like potato, several fruits and wheat. ${ }^{26-29}$ The FFD is not intended as a long-term treatment, but functions as a diagnostic tool to determine whether a child responds to dietary restriction. If a child is responsive to the diagnostic FFD, an individually tailored and more diverse diet can be designed after repeated challenges have identified the foods that trigger ADHD symptoms. ${ }^{30}$

All randomised controlled trials (RCTs) ${ }^{26-29}$ 31-34 applying an FFD in children with ADHD have shown positive effect sizes, ${ }^{20}{ }^{35}$ and according to a meta-analysis by Nigg et al, 33\% of children with ADHD benefited from dietary intervention. ${ }^{36}$ In the most recent RCT, $64 \%$ of a selected subgroup of young children with ADHD responded favourably to the FFD. ${ }^{26}$ However, an FFD is burdensome, and adherence is most often successful in motivated and highly structured families. ${ }^{37}$ For large-scale implementation, simplified dietary treatments should be developed. This requires a detailed understanding of the mechanisms underlying a favourable response to an FFD. However, it is currently unknown how an FFD intervention mediates a decrease of ADHD symptoms in children.

This study will investigate whether an FFD modulates ADHD behaviour through the complex network of molecular communication between the microbiota, gut and brain. Potential candidates are the catecholaminergic neurotransmitters dopamine and norepinephrine, which play an important role in cognitive processes that are often impaired in children with ADHD, such as response inhibition, response conflict and associated error monitoring. ${ }^{38-43}$ Therefore, this study will primarily assess whether the FFD-induced changes in ADHD symptoms are related to changes in (1) neural activation in brain regions related to response inhibition/conflict, ${ }^{44-47}$ (2) functional composition of the gut microbiota related to the metabolism of the dopamine and norepinephrine precursors phenylalanine and tyrosine $e^{48-50}$ and (3) peripheral blood levels of phenylalanine and tyrosine. ${ }^{50}$ Second, a multiomics approach, ${ }^{51-53}$ including profiling of the microbiome, transcriptome, metabolome, methylome and proteome, will be used to unravel the molecular communication in the microbiota-gut-brain (MGB) axis in an unbiased way.

By providing a better understanding of the mechanism(s) that underlie an FFD response, the results of this study can contribute to the development of more effective treatments for $\mathrm{ADHD}$, preventive measures and possibilities for stratification and personalised treatments, that is, starting either treatment as usual or diet therapy, based on the individual's MGB axis configuration.

\section{METHODS AND ANALYSIS}

\section{Study design and registration}

This open-label trial, carried out at Wageningen University \& Research (trial sponsor), will investigate the effects of an FFD on brain function and the MGB axis in relation to changes in ADHD symptoms in right-handed boys with ADHD, aged 8 up to and including 10 years. All participants will follow the FFD. Consequently, parents, children and clinicians cannot be blinded. After screening (T0), eligible participants will start with a 2-week baseline period in which they will maintain and record their regular diet. Thereafter, participants will follow a 5-week FFD preceded by a 1-week transition period. ${ }^{26} 2754$ To improve adherence, parents will receive a diet plan and a recipe book. During the 5-week period, parents and FFD researcher will discuss the child's diet and behaviour regularly (at least once a week). A schematic overview of the study design is provided in figure 1 . Brain function and MGB axis parameters, as well as psychiatric and physical parameters, will be assessed before (T1) and at the end of the 5-week FFD period (T2). An overview of all 


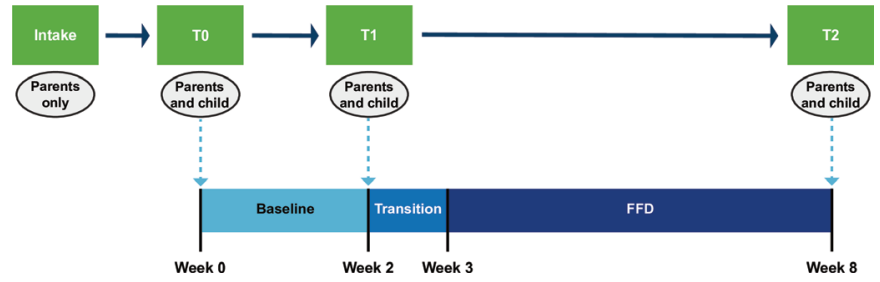

Figure 1 Schematic overview of the BRAIN study design. FFD, few-foods diet. BRAIN, Biomarker Research in ADHD: the Impact of Nutrition.

study parameters and assessment time points is shown in table 1.

This study was registered on ClinicalTrials.gov (number NCT03440346) in February 2018. Due to our misinterpretation of the submission and release procedure, the first informed consent and informed assent forms were unintentionally collected 2 days before the release of the protocol on the website of ClinicalTrials.gov. However, no primary outcome data were collected before the formal release of the study protocol, and no changes in study design or outcome parameter definitions were made between submission and registration date.

\section{Aims and objectives}

1. Primary objectives

a. To investigate whether changes in the metabolism of the dopamine and norepinephrine precursors phenylalanine and tyrosine are related to FFD-induced changes in ADHD symptoms.

b. To investigate whether FFD-induced changes in ADHD symptoms are accompanied by changes in brain activity in functionally related brain regions during task performance, assessing response inhibition/conflict and associated error monitoring.

2. Secondary objectives

a. To explore alternative or synergistic mechanisms underlying the effect of an FFD on ADHD symptoms.

b. To identify biomarkers that predict the response of an individual child to an FFD.

c. To determine whether FFD-induced changes in ADHD symptoms correlate with other physical and behavioural parameters, that is, executive functioning (sustained attention and inhibition), ODD symptoms, social behaviour, physical complaints and stool frequency and type.

\section{Study population}

Boys ( $\geq 8$ and $\leq 10$ years old) meeting the Diagnostic and Statistical Manual of Mental Disorders, fourth edition (DSM-IV) criteria of $\mathrm{ADHD}^{2}$ are considered eligible for inclusion. The lower age limit is set because functional MRI (fMRI) task failure is negligible in children $\geq 8$ years old. ${ }^{55}$ The upper age limit is set because adherence to the FFD can be more successfully achieved in younger children. Due to gender differences ${ }^{56}$ and functional specialisation of the brain in left-handed and right-handed individuals, ${ }^{57}$ the study population is restricted to righthanded boys.

Children who meet at least one of the following criteria are excluded for participation: (1) diagnosis of ASD, developmental coordination disorder, chronic gastrointestinal disorder, autoimmune disorder, dyslexia or dyscalculia, (2) premature birth (<36 weeks) and/or oxygen deprivation during birth, (3) vegetarian/vegan, (4) IQ $<85$, (5) use of systemic antibiotics, antifungals, antivirals or antiparasitics in the past 6 months, ${ }^{58}(6)$ insufficient command of the Dutch language by either parents or child, (7) family circumstances that may compromise following or completion of the diet and (8) having a contraindication to MRI scanning. After T1, participants can be withdrawn or excluded from the trial if the child or family does not comply with the instructions, or if family circumstances interfere with the study compliance.

\section{Primary outcome parameters}

ADHD symptoms

The 18-item ADHD rating scale (ARS) ${ }^{59}$ based on the $\mathrm{DSM}^{-\mathrm{IV}^{2}}$ is the primary outcome measurement of ADHD symptoms in this study. The ARS will be completed by the parents (at T0, T1 and T2), and, if possible, by the child's teacher (at T1 and T2). The ADHD symptoms will be rated using a point-scoring system (0-54 points) ${ }^{26}$ By comparing the scores at $\mathrm{T} 0$ and $\mathrm{T} 1$, the variation in the child's behaviour will be assessed; the difference between the $\mathrm{T} 1$ and $\mathrm{T} 2$ scores will determine the response to the FFD, expressed as a percentage. This percentage will be included in separate analyses as a continuous and as a dichotomous variable (ie, by classifying subjects as FFD responders $(\geq 40 \%$ reduction) or non-responders $(<40 \%$ reduction)).

\section{Neural activation}

As measures of neural activation have been shown to be more robust in demonstrating the nature of response inhibition deficits in ADHD than task performance alone, ${ }^{6061}$ structural and fMRI scans will be conducted during T1 and T2 (at Gelderse Vallei Hospital, Ede, The Netherlands). A structural scan will serve as a reference for further functional scans. Blood oxygen-leveldependent (BOLD) signal changes will be measured while performing cognitive tasks that assess inhibitory control and selective attention, ${ }^{62}$ that is, a stopsignal task (response inhibition) ${ }^{47}$ and a Flanker task (response conflict and associated error monitoring). ${ }^{63}$ fMRI BOLD responses will be assessed between successful and unsuccessful stop or go events (stop task) and between incongruent and congruent events (Flanker task). As a primary activation outcome, effects of the response to the diet will be assessed in functionally defined brain regions of interest (ROI), based on the main effects of tasks across subjects and measurements T1 and T2 (ie, Stop Success >Stop Fail/Go and Incongruent $>$ Congruent). 
Table 1 Overview of MGB axis, ADHD and comorbid parameters

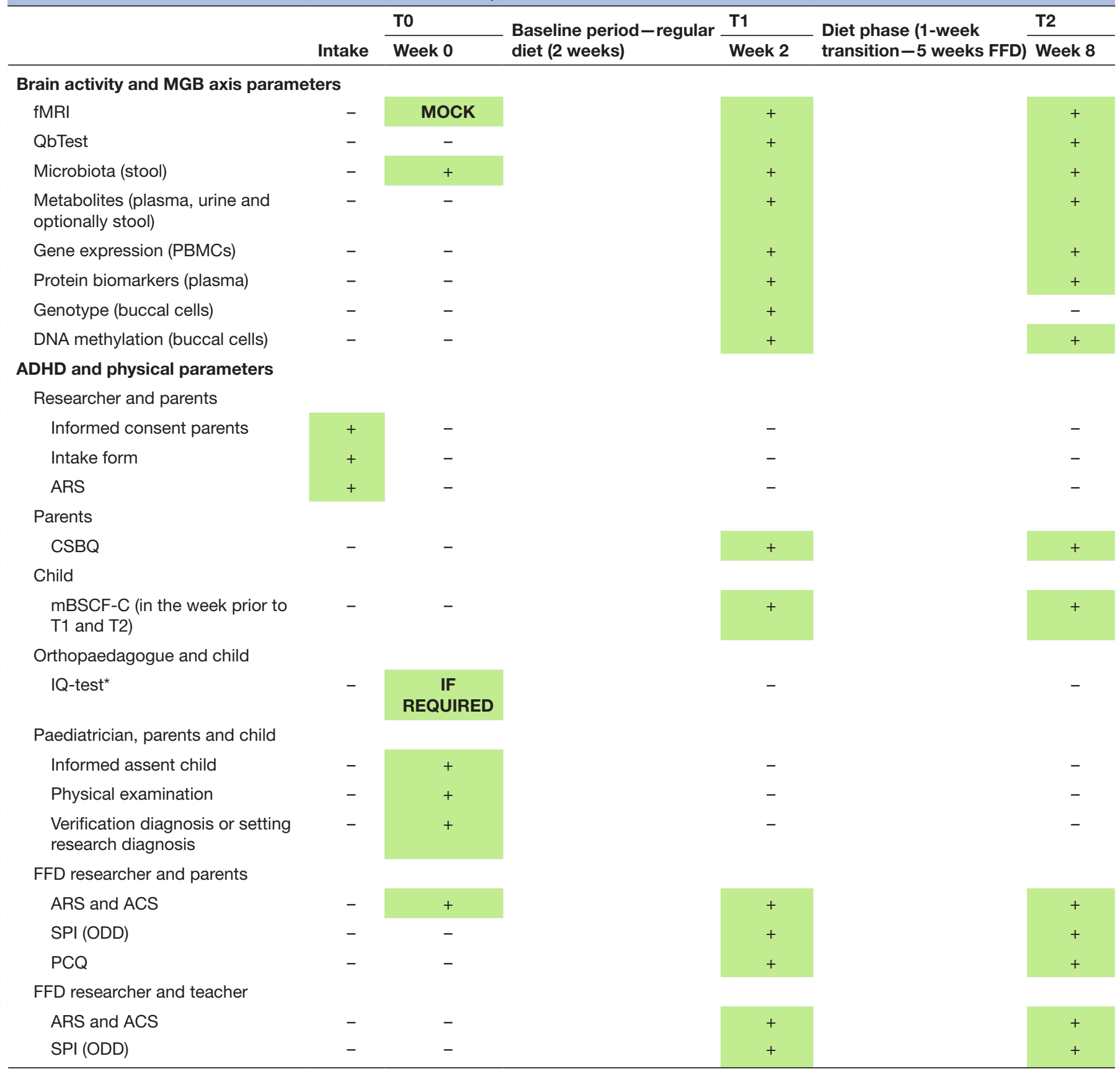

Green: parameter collected; -:, parameter not collected.

*If no IQ test results of the past year are available, an abbreviated IQ test based on the Wechsler Intelligence Scale for Children-III will be conducted. ACS, abbreviated Conners' scale;ADHD, attention deficit hyperactivity disorder; ARS, ADHD Rating Scale; CSBQ, Children's Social and Behavioural Questionnaire;FFD, few-foods diet; fMRI, functional MRI; mBSCF-C, modified Bristol Stool Scale form for Children;MGB, microbiota gut brain; ODD, oppositional defiant disorder; PBMCs, peripheral blood mononuclear cells; PCQ, Physical Complaints Questionnaire; QbTest, quantitative behaviour test; SPI, structured psychiatric interview.

Abundance of genes encoding phenylalanine and tyrosine metabolism enzymes in the gut microbiota

Metagenome profiling will be performed on stool samples collected prior to T1 and T2. The primary focus will be on the abundance of 21 selected microbiota genes that encode enzymes directly involved in the production or degradation of the dopamine and norepinephrine precursors phenylalanine and tyrosine (EG numbers:
1.10.3.1, 1.14.16.1, 1.14.18.1, 1.3.1.43, 1.3.1.78, 1.3.1.79, 1.4.1.20, 1.4.3.2, 2.6.1.1, 2.6.1.5, 2.6.1.57, 2.6.1.58, 2.6.1.9, $4.1 .1 .25,4.1 .1 .28,4.1 .99 .2,4.2 .1 .51,4.2 .1 .91,4.3 .1 .23$, 4.3.1.24, 5.4.3.6; enzyme activities associated with EC numbers can be found at https://www.brenda-enzymes. org/). Abundance profiling will be carried out using HUMAnN2 $2{ }^{64}$ an in silico tool for profiling of microbial genes and biochemical pathways in a community from 
metagenomic sequencing data, or an alternative custom workflow, for example, as described by Yarygin et al. ${ }^{65}$ Pipeline choice will be made prior to data analysis, based on the current state of the art.

\section{Peripheral blood levels of phenylalanine and tyrosine}

Metabolite profiles will be examined in plasma obtained from whole blood and urine (collected at T1 and T2), and analyses will be performed using advanced mass spectrometry. Primary parameters include the levels of phenylalanine and tyrosine in blood. ${ }^{38-42} 50$

\section{Secondary outcome parameters}

Whole brain neural activation patterns

In addition to ROI analyses, task-related fMRI BOLD responses will be assessed using whole brain imaging analyses. ADHD-related differences in functional neural connectivity have been shown in resting state fMRI analyses, ${ }^{66}$ and a reduction in inattentive ADHD symptoms after MPH treatment was associated with changes in resting-state parameters. ${ }^{6768}$ Therefore, resting-state scans will also be performed at $\mathrm{T} 1$ and $\mathrm{T} 2$, to analyse intrinsic brain connectivity and its association with the response to the FFD. To correct for intersubject variability, group analyses (eg, comparisons between responders and nonresponders) will be performed on T2 corrected for T1 per subject.

\section{Executive function assessments}

Executive functioning measurements related to ADHD symptoms, for example, sustained attention and behavioural inhibition, ${ }^{35} 69$ will be conducted during $\mathrm{T} 1$ and T2, using the quantitative behaviour test. ${ }^{69} 70$ This continuous performance test was developed for neuropsychological assessment of ADHD in research and clinical settings and assesses sustained attention, behavioural inhibition and physical movements. The stop-signal ${ }^{441}$ and Flanker tests, ${ }^{63}$ conducted during fMRI and providing information about response times, stop-signal reaction times and commission errors, will also be used for executive functioning analysis.

\section{Related assessments to ADHD, comorbid psychiatric disorders and physical complaints}

In addition to the ARS, the Abbreviated Conners' Scale (ACS) will be used to assess ADHD symptoms. The ACS is frequently used in ADHD research ${ }^{26-29}$ to measure hyperactivity, impulsivity, inattention, mood and temper tantrums. ${ }^{72}$ The maximum total score is 30 ; a total score of more than 15 points is indicative for behavioural problems.

Two comorbid psychiatric disorders, ODD and ASD symptoms, ${ }^{3-5}$ will be assessed during T1 and T2. To assess the ODD symptoms, the eight DSM-IV ${ }^{2}$ criteria that are part of the structured psychiatric interview ${ }^{26}$ will be used; ODD criteria are met when at least four out of eight symptoms occur at least thrice a week. To assess ASD symptoms, the Children's Social and Behavioural Questionnaire $(\mathrm{CSBQ})$ is used. The $\mathrm{CSBQ}$ has been previously used in children with ADHD to assess social behaviour and is applied to measure a wide spectrum of ASD symptoms, including milder, subclinical symptoms in children with ADHD. ${ }^{73-75}$

Many children with ADHD suffer from physical complaints, ${ }^{27-29} 54$ and the FFD can also affect these comorbid physical conditions. ${ }^{29} 54$ Therefore, a Physical Complaints Questionnaire (PCQ $)^{54}$ is taken at $\mathrm{T} 0$ and at T2.

\section{MGB axis parameters}

The taxonomic and functional composition of the microbiota at T1 and T2 will be determined by stool metagenome analysis. By comparing the $\mathrm{T} 0$ and $\mathrm{T} 1$ stool samples, within-participant variation in the gut microbiota can be estimated. Metagenomic DNA sequencing data will be analysed using reference-based methods such as MetaPhlAn2 ${ }^{76}$ and via methods that are part of the CLC Genomics Workbench tool (Qiagen). The functional capacity of the microbiota will be predicted with tools such as HUMAnN2. ${ }^{64}$ 16S rRNA gene sequencing data may be analysed with pipelines such as QIIME ${ }^{77}$ or the CLC Genomics Workbench. Pipeline choice will be made prior to data analysis, based on the current state of the art. In all these analyses, rarefying methods ('subsampling' of sequence reads) will be omitted as these may lead to a loss of power and pose a risk for bias towards false positives. These methods will be replaced by alternative methods, including negative binomial models. ${ }^{78}$

Children with ADHD often suffer from comorbid constipation or faecal incontinence, ${ }^{79}$ which can potentially be relieved by following an FFD. ${ }^{54}$ Since stool frequency and type are dependent on the microbiome composition, ${ }^{80}$ both aspects will be recorded by the child in the week prior to T1 and T2, using the modified Bristol stool scale form for children (mBSCF-C) ${ }^{81}{ }^{82}$ The mBSCF-C, based on the Bristol stool scale form, ${ }^{83}$ consists of five categorical stool consistency types and has been demonstrated to be reliable in a paediatric context. ${ }^{81} 82$

\section{Peripheral blood mononuclear cell gene expression}

Changes in the gene expression profile of peripheral blood mononuclear cells (PBMCs) will be analysed from fasting blood samples collected at T1 and T2. Gene expression profiles will be determined using RNA sequencing.

\section{Metabolite profiling}

Metabolite profiles will be examined in plasma, urine and optionally in stool, collected at T1 and T2. Metabolite analyses will be performed using a pipeline based on advanced mass spectrometry for the accurate quantification of hundreds of metabolites related to amino acid, cofactor and vitamin, nucleotide, carbohydrate, energy and lipid metabolism.

\section{Peripheral blood protein biomarkers}

In peripheral blood, a large panel of proteins, representing neurological, immunological and metabolic markers, will be profiled by implementation of quantitative 
immunoassays or proteomics, using multiplex ELISA approaches. For this analysis, we will use multiplex ELISA panels including chemokines, cytokines, inflammation markers, growth factors and/or metabolic markers.

\section{DNA polymorphisms and genotyping}

Genome-wide profiling of single nucleotide polymorphisms, copy number variants, insertions and deletions will be performed using microarray platforms. Genotyping will be conducted on DNA isolated from buccal cells or PBMCs. Differences in allele frequencies between FFD responders and non-responders will be determined using association analysis in software packages for the analysis of genotyping data such as PLINK. ${ }^{84}$

\section{DNA methylation}

Genome-wide profiling of differential DNA methylation status will be analysed using the Illumina Infinium MethylationEPIC beadchip microarray platform in DNA isolated from buccal cells or PBMCs. DNA from PBMCs is preferred for this analysis. Should the collected material from PBMCs not be sufficient, then DNA from buccal cells will be used for all time points and subjects. This analysis will use samples taken at T1 and T2. Standardised protocols will be used for processing of raw data and downstream data analyses, for example, MethylAid ${ }^{85}$ or RnBeads. ${ }^{86}$ Pipeline choice will be made prior to data analysis, based on the current state of the art.

\section{Sample size calculation}

This study aims to assess the effects of an FFD on brain and MGB axis parameters in relation to ADHD symptoms. FFD responders are defined as children showing at least $40 \%$ reduction on the ARS at T2, compared with T1 ${ }^{26}$ It is assumed that $55 \%$ of the children will respond to the FFD, $25 \%$ will not respond to the FFD ( $\mathrm{n}_{\text {responder }} /$ $\mathrm{n}_{\text {non-responder }}=2.2$ ), and $20 \%$ will drop out. ${ }^{26}$

To our best knowledge, no studies have been published that were conducted on a cohort of ADHD patients and that investigated the effect of an FFD on the brain and MGB axis parameters in relation to ADHD symptoms. Consequently, due to the lack of data on the amplitude and variability in MGB axis parameters induced by an FFD, it is not straightforward to estimate the required sample size to obtain adequate levels of statistical power. Therefore, we based our sample size estimates for primary study outcome parameters on publicly available datasets serving as a proxy for the expected differences and variation.

Sample size calculations were conducted in $G^{*}$ Power $^{87}$ (V.3.1.9.2), using t statistics and comparing two-sample independent means (two tailed). To have a sufficient likelihood of detecting a difference between FFD responders and non-responders for the primary study outcomes, we aim to achieve at least $80 \%$ power with a Bonferroniadjusted error probability of $0.002(0.05 / 25)$ and an outcome allocation ratio $\left(\mathrm{n}_{\text {responder }} / \mathrm{n}_{\text {non-responder }}\right)$ of 2.2 . Based on the sample size estimates per outcome variable, as specified below, we will include 100 participants. Of note, this sample size calculation is conservative, since it is based on a simple Student's t-test comparing the primary measurements between responders and non-responders. The actual analysis (eg, multiple linear regression analysis) will most likely provide additional power.

1. Neural activation: In a study on striatal activation during task performance in boys with ADHD $(n=10$; $8-13$ years) and healthy controls $(\mathrm{n}=6 ; 8-12$ years), activation was higher in controls than in boys with ADHD (Cohen's d: $1.33 \pm 0.13) .{ }^{88}$ The required sample size to detect this difference in striatal activation is $46\left(\mathrm{n}_{\text {respond- }}\right.$ er $=32 ; \mathrm{n}_{\text {non-responder }}=14$ ). We expect a comparable difference between FFD responders and non-responders, as the response to the FFD can be to such extent, that responders no longer meet the criteria for ADHD. ${ }^{26}$

2. Phenylalanine and tyrosine plasma levels: In a study on the effect of a diet intervention trial in 66 healthy adults (31 male and 35 female; 18-70years), baseline plasma metabolites were used to predict outcome (liver dysfunction or no dysfunction; Cohen's d: $1.03 \pm 0.24){ }^{89}$ The required sample size to detect these differences in metabolites is $74\left(\mathrm{n}_{\text {responder }}=51\right.$ and $\left.\mathrm{n}_{\text {non-responder }}=23\right)$.

3. Gene abundance in stool microbiota related to phenylalanine and tyrosine metabolism: As no suitable, publicly available dataset was present at the time of writing this protocol, it was anticipated that gene abundance differences between FFD responders and non-responders will be $25 \%$, with a variance of $25 \%$ (Cohen's d: $1.00 \pm 0.25)$. To detect this difference, a sample size of 70 is required $\left(n_{\text {responder }}=48\right.$ and $\mathrm{n}_{\text {non-responder }}=22$ ).

\section{Statistical analysis}

During initial data processing and primary analyses, the researchers involved in the analysis of laboratory and fMRI data will be blinded to the ADHD symptom scores. On completion of the full data set for all data types, ADHD symptoms scores will be added to reveal responder/nonresponder status for each participant.

To test our primary hypothesis, 25 outcome parameters were selected; neural activation, relative abundance of 21 microbial genes and two plasma metabolites (phenylalanine and tyrosine). Analyses will be conducted using a linear model (analysis of variance (ANOVA)/regression), in which the outcomes at T2 will be analysed in relation to changes in ADHD symptoms (response to FFD, expressed as percentage change and dichotomous), including the values at $\mathrm{T} 1$ as a covariate, in which all positive quantitative variables will be log-transformed. If the regression coefficient for the covariate is (close to) 0 or to -1 , this model shows a direct relation between the outcome at T2 and the change in ADHD symptoms, respectively. Holm-Bonferroni-adjusted $\mathrm{p}$ values will be used based on a family-wise error rate of 0.05 and 25 tests.

Secondary analyses include (1) the analysis of the (multi) omics datasets, (2) the identification of biomarkers that 
can predict responsiveness to the FFD and (3) the analysis of other parameters, such as questionnaires.

i. The omics datasets will first be analysed separately in relation to ADHD symptoms changes using paired statistics (T1 and T2) per child; this way, each child provides his own control data so that effects of interindividual variation will not negatively impact on discriminative analysis power. Next, associations between different (omics)datasets will be examined using multiset analyses. For both types of analysis, we will use constrained ordination methods ${ }^{90}$ with the outcomes at T1 and T2 as 'response data' and the ADHD symptoms change (continuous or dichotomous) as predictor of interest, child as factor covariate and significance tests based on permutation tests (eg, Canoco, ${ }^{91} \operatorname{Vegan}^{92}$ and mixOmics). ${ }^{93}$ For the statistical analysis of count-like compositional data, canonical correspondence analysis can be used. For the statistical analysis of continuous compositional data (with less than $1 \%$ zeroes), weighted Aitchison log-ratio analysis may be applied. ${ }^{94}$ For continuous non-compositional data, constrained principal component analysis can be applied.

ii. Identification of biomarkers that can predict FFD outcome responses will be done via discriminant analyses for microbiota composition data (eg, $\mathrm{LEfSe}^{95}$ and machine learning methods based on Lasso- and Elastic-net (eg, Glmnet, ${ }^{96}$ and/or Random-Forest analysis ${ }^{97}$ to search for correlations between microbiota and omics data.

iii. A linear model (ANOVA/regression) will be applied for the other parameters, for example, ODD, CSBQ and PCQ questionnaires; the outcomes at T2 will be analysed in relation to ADHD symptoms changes, including the values at $\mathrm{T} 1$ as a covariate.

\section{Patient and public involvement}

Patients were indirectly involved in the design of the study by means of collaboration with representatives of patient organisations. In fact, the research question was partly informed by patients' priorities. Many individuals with ADHD are struggling to find a suitable and effective therapy. The FFD has been shown to be very effective in a subset of individuals with ADHD, but this intervention requires a considerable effort of both the child and the parents. Therefore, one of the aims of this study is to find a suitable biomarker that can predict a favourable response to the FFD.

Several participants were actively involved in the recruitment for the study. Some participants shared their experiences with the FFD in a promotional video that leads to the recruitment of other participants. A number of participants were also interviewed by a national newspaper to share their experiences with the Biomarker Research in ADHD: the Impact of Nutrition (BRAIN) study and the FFD. Patients and public will not be involved in the actual conduct of the study. The results will be disseminated to study participants via a newsletter that is sent out four times per year with the most current updates on the study progress and results. If needed (eg, for a medical examination), study participants can request to receive a copy of their personal data. The burden of the diet intervention will be assessed during a weekly telephone interview with the parents of the participants. If the burden is too high, withdrawal from the study will be discussed.

\section{Ethics and dissemination}

The investigators will comply with the principles of the Declaration of Helsinki (adopted by the 18th World Medical Association (WMA) General Assembly, Helsinki, Finland, June 1964, and lastly amended by the 64th WMA General Assembly, Fortaleza, Brazil, October 2013) and with the Medical Research Involving Human Subjects Act. All data collected during the study will be stored in a secure computer database and will be handled confidentially. Chance findings (potentially related to the child's health) will be evaluated by a multidisciplinary committee and communicated to the parents and the general practitioner.

There are no restrictions with respect to publication of the data. The funding institution has no role in study design, data collection, data analysis, data interpretation, writing of the manuscript or in the decision to submit for publication. Both positive and negative results of the study will be made public, preferably as open-access papers in peer-reviewed international scientific journals, according to the Central Committee on Research Involving Human Subjects statement of publication policy. Findings of this study will also be communicated with the general society, for example via presentations in medical centres and through (social) media and the BRAIN study website. The data will be anonymised and made publicly available after publication of results in peer-reviewed scientific journals.

\section{Author affiliations}

${ }^{1}$ Host-Microbe Interactomics, Wageningen University and Research, Wageningen, The Netherlands

${ }^{2}$ Medical Centre Kinderplein, Rotterdam, The Netherlands

${ }^{3} \mathrm{ADHD}$ Research Centre, Eindhoven, The Netherlands

${ }^{4}$ Biometris, Wageningen University and Research, Wageningen, The Netherlands ${ }^{5}$ Donders Centre for Cognitive Neuroimaging, Radboud University, Nijmegen, The Netherlands

${ }^{6}$ Adaptation Physiology, Wageningen University and Research, Wageningen, The Netherlands

Acknowledgements We thank J Matualatupauw, D J Reijngoud, J Toorman, M Führer, T R Licht and M de Boer for valuable input and discussions during the preparation of this protocol.

Contributors LMP initiated the study. LMP, KF, RRP, MK and PvB conceived and designed the study. SPWdV, LMP, RRP, CJFtB, EA, PvB, MK and KF wrote the research protocol. SH and TS wrote the manuscript based on the research protocol, and all authors were involved in editing and approval of the manuscript.

Funding This work was supported by Auxilium Foundation.

Competing interests LMP works at the Pelsser RED Centrum supervising the diagnostic procedure to assess the effect of an FFD on the behaviour of children with ADHD.

Patient consent for publication Not required.

Ethics approval The Medical Research and Ethics Committee of Wageningen University has approved this study (NL63851.081.17, 1 February 2018). 
Provenance and peer review Not commissioned; externally peer reviewed.

Open access This is an open access article distributed in accordance with the Creative Commons Attribution Non Commercial (CC BY-NC 4.0) license, which permits others to distribute, remix, adapt, build upon this work non-commercially, and license their derivative works on different terms, provided the original work is properly cited, appropriate credit is given, any changes made indicated, and the use is non-commercial. See: http://creativecommons.org/licenses/by-nc/4.0/.

ORCID iD

Saartje Hontelez http://orcid.org/0000-0002-5286-7859

\section{REFERENCES}

1 Polanczyk GV, Willcutt EG, Salum GA, et al. Adhd prevalence estimates across three decades: an updated systematic review and meta-regression analysis. Int J Epidemiol 2014;43:434-42.

2 Association AP. Diagnostic and statistical manual of mental disorders. 4th edn. Washington, DC, 2000.

3 Elia J, Ambrosini P, Berrettini W. ADHD characteristics: I. Concurrent co-morbidity patterns in children \& adolescents. Child Adolesc Psychiatry Ment Health 2008;2:15.

4 Jensen CM, Steinhausen H-C. Comorbid mental disorders in children and adolescents with attention-deficit/hyperactivity disorder in a large nationwide study. ADHD Atten Def Hyp Disord 2015;7:27-38.

5 Pliszka SR. Patterns of psychiatric comorbidity with attentiondeficit/hyperactivity disorder. Child Adolesc Psychiatr Clin N Am 2000;9:525-40.

6 Caye A, Swanson J, Thapar A, et al. Life span studies of ADHDConceptual challenges and predictors of persistence and outcome. Curr Psychiatry Rep 2016;18:111.

7 Levy F, Hay DA, McStephen M, et al. Attention-Deficit hyperactivity disorder: a category or a continuum? genetic analysis of a large-scale twin study. J Am Acad Child Adolesc Psychiatry 1997;36:737-44.

8 Akutagava-Martins GC, Rohde LA, Hutz MH. Genetics of attentiondeficit/hyperactivity disorder: an update. Expert Rev Neurother 2016;16:145-56.

9 Hawi Z, Cummins TDR, Tong J, et al. The molecular genetic architecture of attention deficit hyperactivity disorder. Mol Psychiatry 2015;20:289-97.

10 Lee SH, Ripke S, Neale BM, et al. Genetic relationship between five psychiatric disorders estimated from genome-wide SNPs. Nature genetics 2013;45:984-94.

11 Zayats T, Jacobsen KK, Kleppe R, et al. Exome CHIP analyses in adult attention deficit hyperactivity disorder. Trans/ Psychiatry 2016;6:e923.

12 Chen Y-C, Sudre G, Sharp W, et al. Neuroanatomic, epigenetic and genetic differences in monozygotic twins discordant for attention deficit hyperactivity disorder. Mol Psychiatry 2018;23:683-90.

13 Walton E, Pingault J-B, Cecil CAM, et al. Epigenetic profiling of ADHD symptoms trajectories: a prospective, methylome-wide study. Mol Psychiatry 2017;22:250-6.

14 Nigg J, Nikolas M, Burt SA. Measured gene-by-environment interaction in relation to attention-deficit/hyperactivity disorder. $J \mathrm{Am}$ Acad Child Adolesc Psychiatry 2010;49:863-73.

15 Thapar A, Cooper M. Attention deficit hyperactivity disorder. The Lancet 2016;387:1240-50.

16 Excellence NIfHaC. Clinical guideline 72: attention deficit hyperactivity disorder: diagnosis and management of $A D H D$ in children, young people and adults. London: National Institute for Health and Clinical Excellence, 2008. https://www.nice.org.uk/ guidance/cg72

17 Storebø OJ, Ramstad E, Krogh HB, et al. Methylphenidate for children and adolescents with attention deficit hyperactivity disorder (ADHD). Cochrane Database of Systematic Reviews 2015;48.

18 Adler LD, Nierenberg AA. Review of medication adherence in children and adults with ADHD. Postgrad Med 2010;122:184-91.

19 Shaw $M$, Hodgkins $\mathrm{P}$, Caci $\mathrm{H}$, et al. A systematic review and analysis of long-term outcomes in attention deficit hyperactivity disorder: effects of treatment and non-treatment. BMC Med 2012;10:99.

20 Sonuga-Barke EJS, Brandeis D, Cortese S, et al. Nonpharmacological interventions for ADHD: systematic review and meta-analyses of randomized controlled trials of dietary and psychological treatments. Am J Psychiatry 2013;170:275-89.

21 Molina BSG, Hinshaw SP, Swanson JM, et al. The MTA at 8 years: prospective follow-up of children treated for combined-type ADHD in a multisite study. J Am Acad Child Adolesc Psychiatry 2009;48:484-500.
22 Riddle MA, Yershova K, Lazzaretto D, et al. The preschool attentiondeficit/hyperactivity disorder treatment study (PatS) 6-year follow-up. J Am Acad Child Adolesc Psychiatry 2013;52:264-78.

23 van de Loo-Neus GHH, Rommelse N, Buitelaar JK. To stop or not to stop? how long should medication treatment of attention-deficit hyperactivity disorder be extended? European Neuropsychopharmacology 2011;21:584-99.

24 Cox DJ, Moore M, Burket R, et al. Rebound effects with longacting amphetamine or methylphenidate stimulant medication preparations among adolescent male drivers with attentiondeficit/hyperactivity disorder. J Child Adolesc Psychopharmacol 2008;18:1-10.

25 Pelsser LM, Frankena K, Toorman J, et al. Diet and ADHD, reviewing the evidence: a systematic review of meta-analyses of doubleblind placebo-controlled trials evaluating the efficacy of diet interventions on the behavior of children with ADHD. PLoS One 2017;12:e0169277

26 Pelsser LM, Frankena K, Toorman J, et al. Effects of a restricted elimination diet on the behaviour of children with attention-deficit hyperactivity disorder (IncA study): a randomised controlled trial. The Lancet 2011;377:494-503.

27 Pelsser LMJ, Frankena K, Toorman J, et al. A randomised controlled trial into the effects of food on ADHD. Eur Child Adolesc Psychiatry 2009;18:12-19.

28 Carter CM, Urbanowicz M, Hemsley R, et al. Effects of a few food diet in attention deficit disorder. Arch Dis Child 1993;69:564-8.

29 Egger J, Graham PJ, Carter CM, et al. Controlled trial of oligoantigenic treatment in the hyperkinetic syndrome. The Lancet 1985;325:540-5.

30 Heilskov Rytter MJ, Andersen LBB, Houmann T, et al. Diet in the treatment of ADHD in children-A systematic review of the literature. Nord J Psychiatry 2015;69:1-18.

31 Kaplan BJ, McNicol J, Conte RA, et al. Dietary replacement in preschool-aged hyperactive boys. Pediatrics 1989;83:7-17.

32 Boris M, Mandel FS. Foods and additives are common causes of the attention deficit hyperactive disorder in children. Ann Allergy 1994;72:462-8

33 Schulte-Körne G, Deimel W, Gutenbrunner C, et al. [Effect of an oligo-antigen diet on the behavior of hyperkinetic children]. Z Kinder Jugendpsychiatr Psychother 1996;24:176-83.

34 Schmidt $\mathrm{MH}$, Möcks $\mathrm{P}$, Lay $\mathrm{B}$, et al. Does oligoantigenic diet influence hyperactive/conduct-disordered children--a controlled trial. Eur Child Adolesc Psychiatry 1997;6:88-95.

35 Stevenson J, Buitelaar J, Cortese S, et al. Research review: the role of diet in the treatment of attention-deficit/hyperactivity disorder-an appraisal of the evidence on efficacy and recommendations on the design of future studies. J Child Psychol Psychiatry 2014;55:416-27.

36 Nigg JT, Lewis K, Edinger T, et al. Meta-Analysis of attention-deficit/ hyperactivity disorder or attention-deficit/hyperactivity disorder symptoms, restriction diet, and synthetic food color additives. ¡ Am Acad Child Adolesc Psychiatry 2012;51:86-97.

37 Pelsser LM, van Steijn DJ, Frankena K, et al. A randomized controlled pilot study into the effects of a restricted elimination diet on family structure in families with ADHD and odd. Child Adolesc Ment Health 2013;18:39-45

38 Bari A, Mar AC, Theobald DE, et al. Prefrontal and monoaminergic contributions to stop-signal task performance in rats. $J$ Neurosci 2011;31:9254-63.10.1523/JNEUROSCI.1543-11.2011

39 Congdon E, Constable RT, Lesch KP, et al. Influence of SLC6A3 and COMT variation on neural activation during response inhibition. Biol Psychol 2009;81:144-52.

40 Eagle DM, Baunez C. Is there an inhibitory-response-control system in the rat? Evidence from anatomical and pharmacological studies of behavioral inhibition. Neurosci Biobehav Rev 2010;34:50-72.

41 Ghahremani DG, Lee B, Robertson CL, et al. Striatal dopamine D2/D3 receptors mediate response inhibition and related activity in Frontostriatal neural circuitry in humans. $J$ Neurosci 2012;32:7316-24.

42 Rae CL, Nombela C, Rodríguez PV, et al. Atomoxetine restores the response inhibition network in Parkinson's disease. Brain 2016;139:2235-48.

43 Jocham G, Ullsperger M. Neuropharmacology of performance monitoring. Neurosci Biobehav Rev 2009;33:48-60.

44 Rubia K. "Cool" inferior frontostriatal dysfunction in attention-deficit/ hyperactivity disorder versus "hot" ventromedial orbitofrontallimbic dysfunction in conduct disorder: a review. Biol Psychiatry 2011;69:e69-87.

45 Cortese S, Kelly C, Chabernaud C, et al. Toward systems neuroscience of ADHD: a meta-analysis of $55 \mathrm{fMRI}$ studies. Am J Psychiatry 2012;169:1038-55. 
46 Aron AR, Poldrack RA. The cognitive neuroscience of response inhibition: relevance for genetic research in attention-deficit/ hyperactivity disorder. Biol Psychiatry 2005;57:1285-92.

47 Rubia K, Smith AB, Brammer MJ, et al. Right inferior prefrontal cortex mediates response inhibition while mesial prefrontal cortex is responsible for error detection. Neuroimage 2003;20:351-8.

48 Sandhu KV, Sherwin E, Schellekens H, et al. Feeding the microbiotagut-brain axis: diet, microbiome, and neuropsychiatry. Trans/ Res 2017;179:223-44.

49 Fung TC, Olson CA, Hsiao EY. Interactions between the microbiota, immune and nervous systems in health and disease. Nat Neurosci 2017;20:145-55.

50 Aarts E, Ederveen THA, Naaijen J, et al. Gut microbiome in $\mathrm{ADHD}$ and its relation to neural reward anticipation. PLoS One 2017;12:e0183509.

51 Bonder MJ, Tigchelaar EF, Cai X, et al. The influence of a shortterm gluten-free diet on the human gut microbiome. Genome Med 2016;8:45.

52 Gilbert JA, Quinn RA, Debelius J, et al. Microbiome-wide association studies link dynamic microbial consortia to disease. Nature 2016;535:94-103.

53 Hasin Y, Seldin M, Lusis A. Multi-Omics approaches to disease. Genome Biol 2017;18:83.

54 Pelsser LM, Frankena K, Buitelaar JK, et al. Effects of food on physical and sleep complaints in children with ADHD: a randomised controlled pilot study. Eur J Pediatr 2010;169:1129-38.

55 Byars AW, Holland SK, Strawsburg $\mathrm{RH}$, et al. Practical aspects of conducting large-scale functional magnetic resonance imaging studies in children. J Child Neurol 2002;17:885-9.

56 Valera EM, Brown A, Biederman J, et al. Sex differences in the functional neuroanatomy of working memory in adults with ADHD. Am J Psychiatry 2010;167:86-94.

57 Corballis MC. Left brain, right brain: facts and fantasies. PLoS Biol 2014;12:e1001767.

58 Lange K, Buerger M, Stallmach A, et al. Effects of antibiotics on gut microbiota. Dig Dis 2016;34:260-8.

59 DuPaul GJ. Parent and teacher ratings of ADHD symptoms: psychometric properties in a community-based sample. J Clin Child Psychol 1991;20:245-53.

60 van Rooij D, Hoekstra PJ, Mennes M, et al. Distinguishing adolescents with ADHD from their unaffected siblings and healthy comparison subjects by neural activation patterns during response inhibition. Am J Psychiatry 2015;172:674-83

61 Aarts E, van Holstein M, Hoogman M, et al. Reward modulation of cognitive function in adult attention-deficit/hyperactivity disorder: a pilot study on the role of striatal dopamine. Behav Pharmacol 2015;26:227-40.

62 Paloyelis Y, Mehta MA, Kuntsi J, et al. Functional MRI in ADHD: a systematic literature review. Expert Rev Neurother 2007;7:1337-56.

63 Eriksen BA, Eriksen CW. Effects of noise letters upon the identification of a target letter in a nonsearch task. Percept Psychophys 1974;16:143-9.

64 Abubucker S, Segata N, Goll J, et al. Metabolic reconstruction for metagenomic data and its application to the human microbiome. PLoS Comput Biol 2012;8:e1002358.

65 Yarygin K, Tyakht A, Larin A, et al. Abundance profiling of specific gene groups using precomputed gut metagenomes yields novel biological hypotheses. PLoS One 2017;12:e0176154.

66 Castellanos FX, Proal E. Large-Scale brain systems in ADHD: beyond the prefrontal-striatal model. Trends Cogn Sci 2012;16:17-26.

67 Uytun MC, Karakaya E, Oztop DB, et al. Default mode network activity and neuropsychological profile in male children and adolescents with attention deficit hyperactivity disorder and conduct disorder. Brain Imaging Behav 2017;11:1561-70.

68 Yoo JH, Kim D, Choi J, et al. Treatment effect of methylphenidate on intrinsic functional brain network in medication-naïve ADHD children: a multivariate analysis. Brain Imaging Behav 2018;12:518-31.

69 Hall CL, Valentine AZ, Walker GM, et al. Study of user experience of an objective test (QbTest) to aid ADHD assessment and medication management: a multi-methods approach. BMC Psychiatry 2017;17:66.

70 Reh V, Schmidt M, Lam L, et al. Behavioral assessment of core ADHD symptoms using the QbTest. J Atten Disord 2015;19:1034-45.
71 Zandbelt BB, Vink M. On the role of the striatum in response inhibition. PLoS One 2010;5:e13848.

72 Conners CK, Goyette CH, Southwick DA, et al. Food additives and hyperkinesis: a controlled double-blind experiment. Pediatrics 1976;58:154-66.

73 Hartman CA, Luteijn E, Serra M, et al. Refinement of the children's social behavior questionnaire (CSBQ): an instrument that describes the diverse problems seen in milder forms of PDD. J Autism Dev Disord 2006;36:325-42.

74 Luteijn EF, Serra M, Jackson S, et al. How unspecified are disorders of children with a pervasive developmental disorder not otherwise specified? A study of social problems in children with PDD-NOS and ADHD. Eur Child Adolesc Psychiatry 2000;9:168-79.

75 O'Dwyer L, Tanner C, van Dongen EV, et al. Brain volumetric correlates of autism spectrum disorder symptoms in attention deficit hyperactivity disorder. PLoS One 2014;9:e101130.

76 Truong DT, Franzosa EA, Tickle TL, et al. MetaPhIAn2 for enhanced metagenomic taxonomic profiling. Nat Methods 2015;12:902-3.

77 Caporaso JG, Kuczynski J, Stombaugh J, et al. QIIME allows analysis of high-throughput community sequencing data. Nat Methods 2010;7:335-6.

78 McMurdie PJ, Holmes S. Waste not, want not: why rarefying microbiome data is inadmissible. PLoS Comput Biol 2014;10:e1003531.

79 McKeown C, Hisle-Gorman E, Eide M, et al. Association of constipation and fecal incontinence with attention-deficit/ hyperactivity disorder. Pediatrics 2013;132:e1210-5.

80 Vandeputte D, Falony G, Vieira-Silva S, et al. Stool consistency is strongly associated with gut microbiota richness and composition, enterotypes and bacterial growth rates. Gut 2016;65:57-62.

81 Chumpitazi BP, Lane MM, Czyzewski DI, et al. Creation and initial evaluation of a stool form scale for children. J Pediatr 2010;157:594-7

82 Lane MM, Czyzewski DI, Chumpitazi BP, et al. Reliability and validity of a modified Bristol stool form scale for children. $J$ Pediatr 2011;159:437-41.

83 Lewis SJ, Heaton KW. Stool form scale as a useful guide to intestinal transit time. Scand J Gastroenterol 1997;32:920-4

84 Daneshjou R, Zappala Z, Kukurba K, et al. PATH-SCAN: a reporting tool for identifying clinically actionable variants. Pac Symp Biocomput 2014:229-40.

85 van Iterson M, Tobi EW, Slieker RC, et al. MethylAid: visual and interactive quality control of large Illumina 450k datasets. Bioinformatics 2014;30:3435-7.

86 Assenov Y, Müller F, Lutsik P, et al. Comprehensive analysis of DNA methylation data with RnBeads. Nat Methods 2014;11:1138-40.

87 Faul F, Erdfelder E, Buchner A, et al. Statistical power analyses using G*Power 3.1: tests for correlation and regression analyses. Behav Res Methods 2009;41:1149-60.

88 Vaidya CJ, Austin G, Kirkorian G, et al. Selective effects of methylphenidate in attention deficit hyperactivity disorder: a functional magnetic resonance study. Proc Natl Acad Sci U S A 1998;95:14494-9.

89 Sha W, da Costa K-A, Fischer LM, et al. Metabolomic profiling can predict which humans will develop liver dysfunction when deprived of dietary choline. Faseb J 2010;24:2962-75.

90 ter Braak CJF, Prentice IC. A theory of gradient analysis. Adv Ecol Res 1988;34:235-82.

91 ter Braak CJF, milauer P. Canoco reference manual and user's guide: software for ordination, version 5.0. 496. Ithaca USA: Microcomputer Power, 2012

92 Dixon P. Vegan, a package of $\mathrm{R}$ functions for community ecology. $J$ Veg Sci 2003;14:927-30.

93 Rohart F, Gautier B, Singh A, et al. mixOmics: An R package for 'omics feature selection and multiple data integration. PLOS Comput Biol 2017;13:e1005752.

94 Greenacre M. Log-Ratio Analysis Is a Limiting Case of Correspondence Analysis. Math Geosci 2010;42:129-34.

95 Segata N, Izard J, Waldron L, et al. Metagenomic biomarker discovery and explanation. Genome Biol 2011;12:R60.

96 Friedman J, Hastie T, Tibshirani R. Regularization paths for generalized linear models via coordinate descent. J Stat Softw 2010;33:1-22.

97 Breiman L. Random forests. Mach Learn 2001;45:5-32. 